\title{
LASER ASSISTED HATCHING FOR HUMAN CRYOPRESERVED CLEAVED EMBRYOS
}

\author{
Kenichiro Hiraoka ${ }^{1}$, Kaori Hiraoka ${ }^{1}$, Miyuki Miyazaki ${ }^{1}$, Emi Fukunaga ${ }^{1}$ \\ Toshitaka Horiuchi ${ }^{2}$, Tomoyo Kusuda ${ }^{1}$, Shinichiro Okano ${ }^{1}$ \\ Masayuki Kinutani ${ }^{1}$ and Kazuo Kinutani ${ }^{1}$ \\ 1: Kinutani Women's Clinic \\ 2: Graduate School of Applied Biosciences, Hiroshima Prefectural University
}

\begin{abstract}
The objectives of this study were to evaluate the impact of two different sizes of zona pellucida thinning area by laser assisted hatching on the clinical outcome of cryopreserved cleaved embryos (Experiment 1) and two different sizes of zona pellucida opening area by laser assisted hatching on the clinical outcome of cryopreserved cleaved embryos that were cultured to blastocyst (Experiment 2). A total of 120 cryopreserved cleaved embryo transfers were assigned to either quarter or half of zona pellucida thinning group. Laser assisted hatching was conducted at the cleavage-stage. The rates of clinical pregnancy ( 47 versus $25 \%$ ) and implantation (32 versus 16\%) were significantly greater in the half thinning group than in the quarter thinning group $(\mathrm{P}=0.0218$ and $\mathrm{P}=0.0090$, respectively) (Experiment 1 ). A total of 71 cryopreserved cleaved embryo transfers were assigned to either one eighth or half of zona pellucida opening group. The cryopreserved cleaved embryos were cultured to blastocyst and laser assisted hatching was conducted at the blastocyst-stage. The rates of clinical pregnancy (74 versus 43\%) and implantation (52 versus 27\%) were significantly greater in the half opening group than in the one eighth opening group $(\mathrm{P}=$ 0.0090 and $\mathrm{P}=0.0117$, respectively) (Experiment 2). Our results suggest that the size of zona pellucida thinning area or opening area can affect the clinical outcome of cryopreserved cleaved embryo transfers.
\end{abstract}

Key Words: assisted reproductive technology, human embryo, laser assisted hatching

\section{Introduction}

Assisted reproductive technology generally produces surplus embryos that can be cryopreserved for later use. The success of a cryopreservation programme will undoubtedly increase the cumulative pregnancy rates. However, success associated with the transfer of cryopreserved embryos has been generally lower than that obtained with fresh embryo transfer ${ }^{1}$ ). One explanation may be due to zona pellucida hardening during the cryopreservation process. Therefore, an artificial thinning or opening in the zona pellucida, i.e. assisted hatching, may be useful for cryopreserved embryo

Addressee for Correspondence:

Kenichiro Hiraoka

Kinutani Women's Clinic, 8-23-4F, Hondori, Naka-ku,

Hiroshima 730-0035, Japan

Tel: +81 82 2476399; FAX: +81 822478903

E-mail: hiraoka@chive.ocn.ne.jp transfer. Recently, assisted hatching by $1.48 \mu \mathrm{m}$ diode laser has been introduced and has enabled to reliably produce equally sized zona pellucida thinning or opening ${ }^{2}$. However, studies evaluating the effect of the size of zona pellucida thinning area or opening area for cryopreserved embryos by laser assisted hatching are lacking. The objectives of this study were to evaluate the results of two different sizes (quarter or half) of zona pellucida thinning area by laser assisted hatching for cryopreserved cleaved embryos (Experiment 1) and two different sizes (one eighth or half) of zona pellucida opening area by laser assisted hatching for cryopreserved cleaved embryos that were cultured to blastocyst (Experiment 2).

Manuscript received: December 7th, 2009

Accepted for publication: February 8th, 2010 


\section{ORIGINAL ARTICLES}

\section{Materials and Methods}

\section{Patients}

\section{Experiment 1}

This study consisted of 120 patients of cryopreserved cleaved embryo transfers conducted at Kinutani Women's Clinic between February 2005 and September 2008. On the day of cryopreserved cleaved embryo transfer, patients were randomized by a laboratory technician according to a computer-generated randomization list in sealed envelopes into the quarter of zona pellucida thinning group or the half of zona pellucida thinning group. Both patients and clinicians were unaware of the group assignments until the completion of the study.

\section{Experiment 2}

This study consisted of 71 patients of cryopreserved cleaved embryo transfers conducted at Kinutani Women's Clinic between October 2003 and June 2006. The cryopreserved cleaved embryos were thawed and cultured to blastocyst. One eighth opening of the zona pellucida was performed for 40 patients between October 2003 and January 2005 (one eighth opening group). Half of the zona pellucida opening was performed for 31 patients between February 2005 and June 2006 (half opening group).

We do not have Institutional Review Board in our private clinic. Drs K. Kinutani, M. Kinutani, S. Okano and T. Kusuda are members of Japan Society of Obstetrics and Gynecology (JSOG) and Kinutani Women's Clinic have been registered as certified fertility centres by JSOG. These studies were conducted according to the guidelines of JSOG. Furthermore, each couple included in these studies was asked to sign an approval consent form before enrolling in the study.

\section{IVF procedure}

Women were treated with gonadotrophin-releasing hormone $(\mathrm{GnRH})$ analogue buserelin acetate (Mochida, Tokyo, Japan) from either the preceding mid-luteal phase in a long treatment protocol or second day of the cycle in a short treatment protocol. Ovarian stimulation was carried out with human menopausal gonadotrophin (Nikken, Tokyo, Japan) or urinary FSH (Fertinorm; Serono, Japan). Follicular development was monitored with serial (every third day) vaginal ultrasound examinations and serum oestradiol measurements. Human chorionic gonadotrophin (HCG; Teizo, Tokyo, Japan) was administered to women when dom- inant follicles reached a diameter of $18 \mathrm{~mm}$. Oocytes were collected $35 \mathrm{~h}$ after HCG administration using a vaginal ultrasound-guided procedure and were incubated in human tubal fluid (HTF) medium (Irvine Scientific, CA, USA) containing 10\% (v/v) serum substitute supplement (SSS; Irvine). Sperm preparation was carried out using discontinuous ISolate ${ }^{\mathrm{TM}}$ (Irvine) gradient. Mature oocytes were either inseminated with spermatozoa 5-7 h after oocyte retrieval at a concentration of 100,000 to 200,000 motile spermatozoa $/ \mathrm{ml}$ for 5 to 10 oocytes (cIVF) or microinjected with a single spermatozoon (ICSI). The day of oocyte retrieval was considered as day 0. Fertilization was confirmed at 24-25 h after oocyte retrieval (day 1) by the presence of two pronuclei.

Fertilized oocytes were transferred and cultured in P-1 (Irvine) containing 10\% (v/v) SSS (Irvine) from day 1 to day 3. All oocytes and embryos were incubated at $37{ }^{\circ} \mathrm{C}$ in an atmosphere of $6 \% \mathrm{CO} 2,5 \% \mathrm{O} 2$ and $89 \%$ N2. Fresh embryos were transferred on day 2 or day 3. The day of embryo transfer and vitrified were determined according to the clinical or patient's schedules. In our previous clinical experience, the rates of pregnancy and implantation after fresh or cryopreserved day 2 and day 3 transfers were similar. Therefore, in the present study, the results of cryopreserved day 2 and day 3 transfers were analyzed together. Some patients abandoned fresh embryo transfer in order to avoid potential risks of ovarian hyperstimulation syndrome. Good quality embryos were defined as those having regular blastomeres, $<20 \%$ fragments and no multinucleated blastomeres and those containing at least three cells on day 2 or six cells on day 3 . Embryos that in addition had cleaved early were considered top quality embryos, and were first choice for fresh transfer. After the transfer of fresh embryos, all surplus embryos were cryopreserved on day 2 or day 3 only for patients who had at least 1 good quality embryo in their surplus embryos.

\section{Cryopreservation of embryos}

\section{Experiment 1}

The embryos were vitrified by the method developed

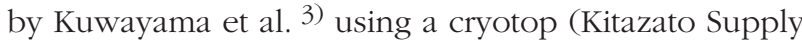
Co., Fujinomiya, Japan), albeit with slight modifications, and has been described previously ${ }^{4)}$. The cryotop consists of a $0.4 \mathrm{~mm}$ wide $\times 20 \mathrm{~mm}$ long $\times 0.1$ $\mathrm{mm}$ thick polypropylene strips attached to a plastic handle and equipped with a cover straw. As the base medium, modified HTF medium-HEPES (Irvine) plus $20 \%(\mathrm{v} / \mathrm{v})$ SSS (Irvine) was used. The equilibration solution contained 7.5\% (v/v) ethylene glycol (Sigma 
Chemical Co., MO, USA) and 7.5\% (v/v) dimethyl sulphoxide (Kanto Chemical Co., Tokyo, Japan). The vitrification solution was composed of $15 \%(\mathrm{v} / \mathrm{v})$ ethylene glycol, 15\% (v/v) dimethyl sulphoxide and 0.5 mol/1 sucrose (Nacalai Tesque, Inc., Kyoto, Japan). The embryos were incubated in $1 \mathrm{ml}$ of $30^{\circ} \mathrm{C}$ equilibration solution for $10 \mathrm{~min}$, confirming shrinkage and reexpansion before exposure to the vitrification solution. After equilibration, the embryos were then incubated in $1 \mathrm{ml}$ of $30^{\circ} \mathrm{C}$ vitrification solution and loaded, within $45 \mathrm{~s}$, onto the tip of the cryotop with $\sim 1 \mu \mathrm{l}$ of cryoprotectant solution. Then the cryotop was immediately submerged into liquid nitrogen and under the liquid nitrogen the plastic cover was placed over the strip to provide protection during storage.

Vitrified embryos considered top quality in each patient's vitrified embryos were warmed on the day of embryo transfer. The warming procedure was done as follows. The protective cover was removed in liquid nitrogen and the end of the polypropylene strip was immersed directly into $1 \mathrm{ml}$ of $37^{\circ} \mathrm{C} 1.0 \mathrm{~mol} / 1$ sucrose solution for $1 \mathrm{~min}$. The embryos were then transferred into $1 \mathrm{ml}$ of $37^{\circ} \mathrm{C} 0.5 \mathrm{~mol} / 1$ sucrose solution for $3 \mathrm{~min}$ and washed twice in the base medium for $5 \mathrm{~min}$. After worming, vitrified embryos were examined the total number of visible blastomeres and the presence of lysed blastomeres, and the embryos with more than $75 \%$ of visible blastomeres were judged to have survived.

\section{Experiment 2}

The embryos were cryopreserved using a programmable freezer (FREEZE CONTROL ${ }^{\circledR}$ MODEL CL-863, CryoLogic Pty Ltd, Australia). The embryos were cryopreserved using Dulbecco's phosphate buffered saline solution (PBS 1X; Irvine) supplemented with 20\% (v/v) SSS (Irvine), $0.1 \mathrm{~mol} / 1$ sucrose and $1.4 \mathrm{~mol} / 1$ ethylene glycol. The freezing program for embryos in our clinic was as follows: starting temperature: $20^{\circ} \mathrm{C}$; rate of cooling: $2^{\circ} \mathrm{C} / \mathrm{min}$ from 20 to $-7^{\circ} \mathrm{C}$; soak at $-7^{\circ} \mathrm{C}$ for $5 \mathrm{~min}$; manual seeding; hold the temperature at $-7^{\circ} \mathrm{C}$ for 10 min; rate of cooling: $0.3^{\circ} \mathrm{C} / \mathrm{min}$ from -7 to $-32^{\circ} \mathrm{C}$; rate of cooling: $4^{\circ} \mathrm{C} / \mathrm{min}$ from -32 to $-86^{\circ} \mathrm{C}$. The frozen straw was quickly transferred from freezing chamber to a reservoir of liquid nitrogen.

Thawing procedure was done as follows. Frozen embryos were thawed at room temperature for $40 \mathrm{~s}$ and then at $30^{\circ} \mathrm{C}$ in a water bath for $40 \mathrm{~s}$ Subsequently, the cryoprotectant was removed by washing the embryos successively with a decreasing concentration of ethylene glycol. After thawing, frozen embryos were examined the total number of visible blastomeres and the presence of lysed blastomeres, and the embryos with more than $75 \%$ of visible blastomeres were judged to have survived. For all the couples, all the remaining frozen day 2 or day 3 embryos were thawed and cultured for better embryo selection before embryo transfer. Following thawing, thawed day 2 (the day of thawing was considered as day 2) embryos were placed in Blast Assist Medium 1 (Medicult, Denmark), in Blast Assist Medium 2 (Medicult) on day 3 and cultured until day 6; thawed day 3 (the day of thawing was considered as day 3) embryos were placed in Blast Assist Medium 2 (Medicult) and cultured until day 6 at $37^{\circ} \mathrm{C}$ in an atmosphere of $6 \% \mathrm{CO}_{2}, 5 \% \mathrm{O} 2$ and $89 \% \mathrm{~N} 2$. On day 5, the inner cell mass (ICM) and trophectoderm (TE) of the blastocyst was examined. The ICM grading was as follows: A. tightly packed, many cells; B. loosely grouped, several cells; C. very few cells. The TE grading was as follows: A. many cells forming a tightly knit epithelium; B. few cells; C. very few cells forming a loose epithelium ${ }^{5)}$. In all cases only day 5 expanded blastocysts which were not hatching or had not hatched from the zona pellucida and scoring $\mathrm{BB}$ or higher were transferred (Figure 2a).

\section{Assisted hatching methods}

\section{Experiment 1}

As soon as warming of embryos was completed, assisted hatching was performed by the method as described previously, albeit with slight modifications ${ }^{4)}$. Warmed embryos were placed under mineral oil within a $50 \mu 1$ microdroplet of $37^{\circ} \mathrm{C}$ Sperm Washing Medium (Irvine) in a Petri dish and positioned on the phase-contrast inverted microscope stage.

Quarter or half of zona pellucida thinning using laser (Zilos-tk Laser, Hamilton Thorne Research, Beverly, MA, USA) was performed as follows. Embryos were stabilized with a holding pipette held at the 9 o' clock position (Figure 1a), and positioned with the laser target located on the outer edge of the zona pellucida. The power of laser was $100 \%$ and the pulse duration was $300 \mu \mathrm{s}$. By this setting, a $5 \mu \mathrm{m}$ hole was formed in the zona pellucida by one laser shot. Quarter or half of zona pellucida thinning was performed by thinning the zona pellucida at a depth of $50-80 \%$ of the zona pellucida thickness, estimated from an area of empty perivitelline space, initiated at one point and continued until quarter or half of the zona pellucida was irradiated, i.e. laser thinning was initiated at the 12 o'clock position and consecutive irradiations were generated until the 3 o'clock position (quarter thinning) (Figure 1b) or the 6 o'clock position 


\section{ORIGINAL ARTICLES}
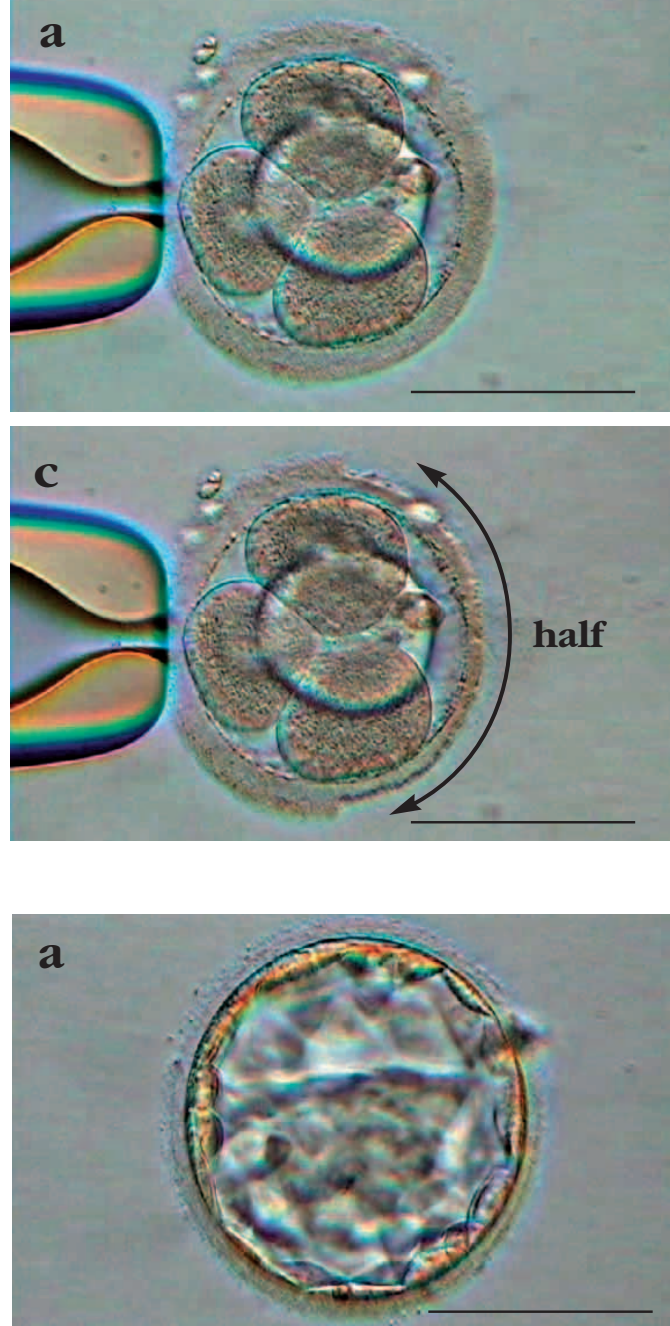

Fig. 2: A human blastocyst developed from frozen cleaved embryo (a), after immersed into $0.2 \mathrm{~mol} / 1$ sucrose solution for $2 \mathrm{~min}$ (b). Bar represents $100 \mu \mathrm{m}$.
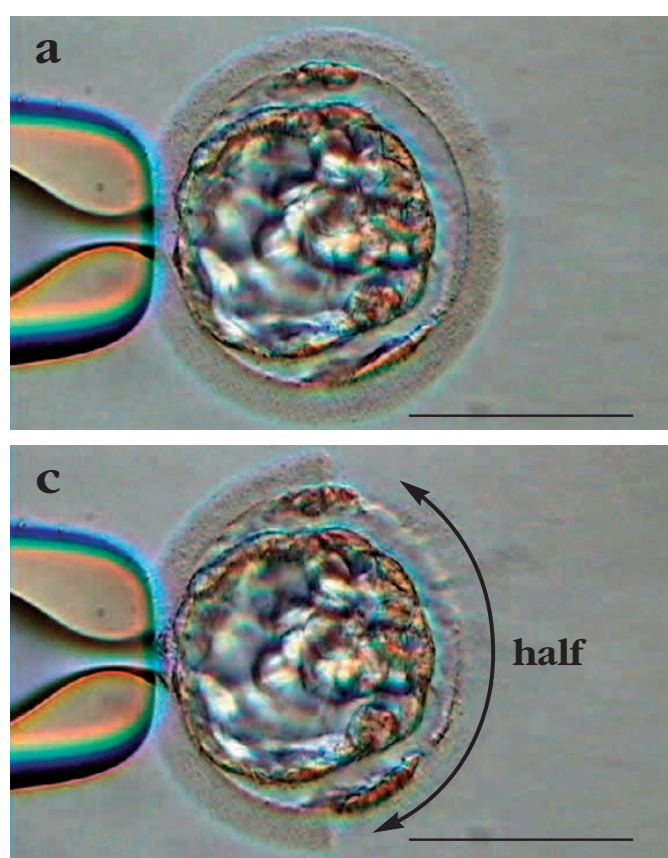
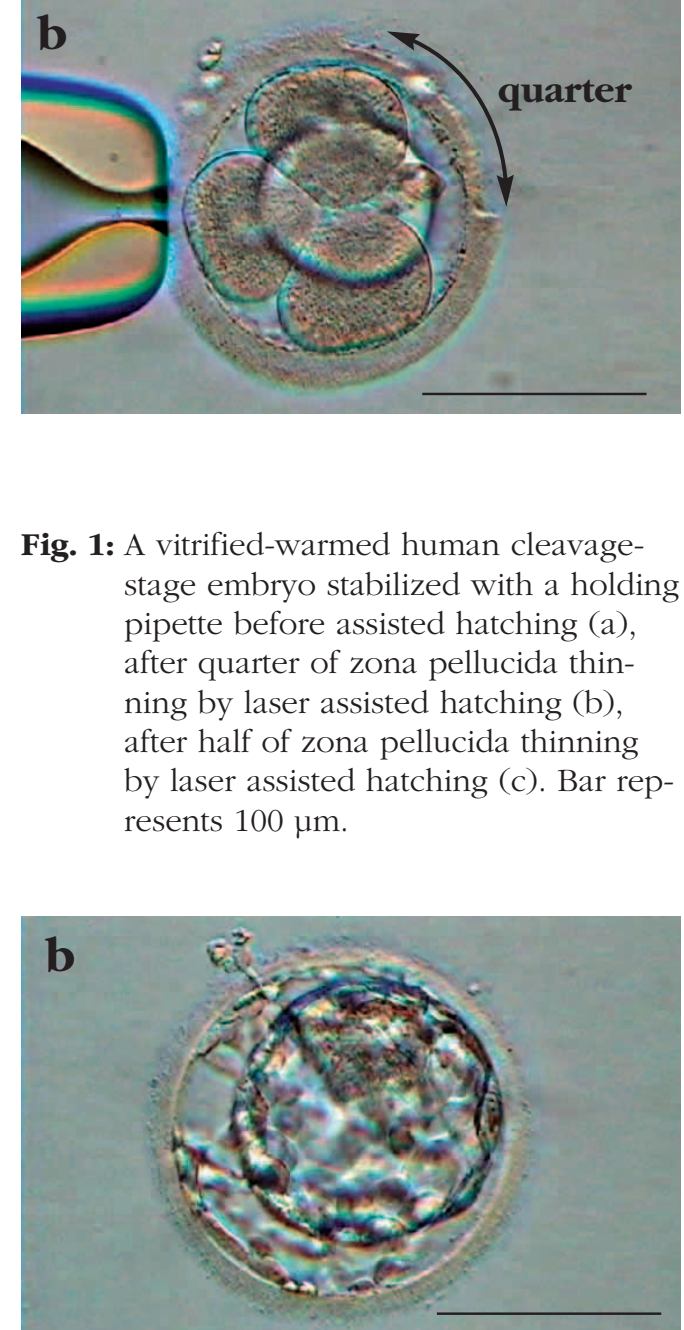

Fig. 1: A vitrified-warmed human cleavagestage embryo stabilized with a holding pipette before assisted hatching (a), after quarter of zona pellucida thinning by laser assisted hatching (b), after half of zona pellucida thinning by laser assisted hatching (c). Bar represents $100 \mu \mathrm{m}$.

Fig. 3: A human blastocyst developed from frozen cleaved embryo stabilized with a holding pipette before assisted hatching (a), after one eighth of zona pellucida opening by laser assisted hatching (b), after half of zona pellucida opening by laser assisted hatching(c). Bar represents $100 \mu \mathrm{m}$. 
(half thinning) (Figure 1c) was reached. After the assisted hatching procedure was completed, the embryos were rinsed several times and were cultured in P-1 (Irvine) containing 10\% (v/v) SSS (Irvine) for $3 \mathrm{~h}$ until transfer.

\section{Experiment 2}

Assisted hatching was performed at the expanded blastocyst stage (Figure 2a) by the method as described previously ${ }^{6}$ ). Before assisted hatching, the blastocysts were placed into $1.0 \mathrm{ml}$ of $25^{\circ} \mathrm{C} 0.2 \mathrm{~mol} / 1$ sucrose in Sperm Washing Medium (Irvine) to shrink the blastocyst away from the zona pellucida allowing the hole to be opened through the zona pellucida without potential harm to the embryo itself (Figure 2b).

One eighth or half of the zona pellucida opening assisted hatching using laser (Zilos-tk Laser) was performed as follows. Embryos were stabilized with a holding pipette held at the 9 o'clock position (Figure $3 \mathrm{a}$ ), and positioned with the laser target located on the outer edge of the zona pellucida. The power of laser was $100 \%$ and the pulse duration was $500 \mu$ s. By using this setting, a $10 \mu \mathrm{m}$ hole was formed in the zona pellucida by one laser shot. Multiple irradiations along the convex periphery of zona pellucida from outward to inward were used to form a $10 \mu \mathrm{m}$ opening. Similarly, another opening was formed next to the first one. This procedure was repeated until one eighth opening (about $40 \mu \mathrm{m}$ ) (Figure 3b) or half of the zona pellucida opening was formed. The opening of half of the zona pellucida was initiated at one point and continued until half of the zona pellucida was opened (e.g. laser opening was initiated at the 12 o'clock position, and consecutive shots were applied until the 6 o'clock position of the embryo was reached) (Figure 3c). After the assisted hatching procedure was completed, the blastocysts were rinsed several times and were cultured in Blast Assist Medium 2 (Medicult) for $3 \mathrm{~h}$ until transfer.

\section{Endometrial preparation}

Cryopreserved embryo transfer was performed in hormonal replacement treatment cycles. All women received transdermal oestradiol (Estraderm 1.4-5.8 mg, Kissei, Tokyo, Japan) with gonadotrophin-releasing hormone analogue for the preparation of the endometrium. The administration of progesterone (vaginal $400 \mathrm{mg}$ daily) was initiated when endometrial thickness exceeded $10 \mathrm{~mm}$. In experiment 1, cryopreserved day 2 and day 3 embryo transfers were scheduled on day 2 and on day 3 after the initiation of progesterone treatment respectively. In experiment 2 , embryo transfer was scheduled on day 5 after the initiation of progesterone treatment. One to two embryos were transferred into the patient's uterus.

\section{Assessment of pregnancy}

A clinical pregnancy was assessed by a positive fetal heartbeat on transvaginal ultrasound at 5-6 weeks of pregnancy. The implantation rate was calculated as the number of gestational sacs identified on transvaginal ultrasound per number of cryopreserved transferred embryos.

\section{Statistical analysis}

The Mann-Whitney test, unpaired Student's t test, chisquared test and Fisher's exact test were used as appropriate to determine statistical differences between groups. A $P$ value of $<0.05$ was considered significant.

\section{Results}

\section{Experiment 1}

There were no differences between the quarter thinning and half thinning groups in the age of women at vitrification and warming, primary infertility, secondary infertility, cause of infertility, the number of IVF attempts, the number of oocytes, the number of metaphase II oocytes, the percentage of cIVF or ICSI cycle and the number of embryos. The number of embryos transferred, clinical pregnancy rate, implantation rate, twin pregnancy rate, delivery rate, the number of embryos vitrified, the distribution between day 2 and day 3 embryos vitrified and the distribution between good quality day 2 and day 3 embryos vitrified after fresh embryo transfer were also comparable between the two groups (data not shown).

Table 1 summarizes the outcomes of embryo transfer. The distribution between day 2 and day 3 embryos warmed, the distribution between good quality day 2 and day 3 embryos warmed, survival rate and the number of embryos transferred were similar between the two groups. On the other hand, clinical pregnancy and implantation rates were significantly higher in the half thinning group compared with the quarter thinning group ( $47 \%$ versus $25 \%, \mathrm{P}=0.0218$; $32 \%$ versus $16 \%, \mathrm{P}=0.0090)$. No differences in the twin pregnancy and miscarriage rates per clinical pregnancy were found between the two groups.

\section{Experiment 2}

There were no differences between the one eighth opening and half opening groups in the age of women at freezing and thawing, primary infertility, secondary 


\section{ORIGINAL ARTICLES}

Table 1. Comparison of outcomes of cryopreserved cleaved embryos between the quarter of zona pellucida thinning and the half of zona pellucida thinning groups.

\begin{tabular}{|c|c|c|c|}
\hline Parameter & quarter $(n=60)$ & half $(n=60)$ & P-value \\
\hline No. of embryos warmed & 104 & 107 & - \\
\hline No. of day 2/day 3 embryos warmed & $48 / 56$ & $54 / 53$ & NS \\
\hline No. of good quality embryos warmed & 65 & 61 & NS \\
\hline No. of good quality day $2 /$ day 3 embryos warmed & $28 / 37$ & $27 / 34$ & NS \\
\hline No. of embryos survived (\%) & $99(95)$ & $103(96)$ & NS \\
\hline Mean no. of embryos transferred \pm SD & $1.7 \pm 0.5$ & $1.7 \pm 0.6$ & NS \\
\hline No. of clinical pregnancies (\%) & $15(25)$ & $28(47)$ & 0.0218 \\
\hline No. of embryos implanted (\%) & $16(16)$ & $33(32)$ & 0.0090 \\
\hline No. of twin pregnancies (\%) & $1(7)$ & $5(18)$ & NS \\
\hline No. of miscarriages (\%) & $2(13)$ & $3(11)$ & NS \\
\hline
\end{tabular}

NS = not significant. Good quality embryos were defined as those having regular blastomeres,

$<20 \%$ fragments and no multinucleated blastomeres and those containing at least three cells on day 2 or six cells on day 3 .

Table 2. Comparison of outcomes of cryopreserved cleaved embryos that were culture to blastocyst between the one eighth of zona pellucida opening and the half of zona pellucida opening groups.

\begin{tabular}{|c|c|c|c|}
\hline Parameter & one eighth $(n=40)$ & half $(n=31)$ & P-value \\
\hline No. of blastocysts transferred & 62 & 52 & - \\
\hline \multicolumn{4}{|l|}{ Morphology scores of blastocysts } \\
\hline $\mathrm{AA}(\%)$ & $25(40)$ & $20(38)$ & NS \\
\hline $\mathrm{AB}(\%)$ & $12(20)$ & $10(19)$ & NS \\
\hline $\mathrm{BB}(\%)$ & $25(40)$ & $22(42)$ & NS \\
\hline Mean no. of embryos transferred \pm SD & $1.6 \pm 0.5$ & $1.7 \pm 0.5$ & NS \\
\hline No. of clinical pregnancies (\%) & $17(43)$ & $23(74)$ & 0.0090 \\
\hline No. of embryos implanted (\%) & $17(27)$ & $27(52)$ & 0.0117 \\
\hline No. of twin pregnancies (\%) & $0(0)$ & $4(17)$ & NS \\
\hline No. of miscarriages (\%) & $2(12)$ & $3(13)$ & NS \\
\hline
\end{tabular}

NS = not significant. Morphology scores of blastocysts: e.g. AA = inner cell mass grading was A

and trophectoderm grading was A, for details see Materials and Methods.

infertility, cause of infertility, the number of IVF attempts, the number of oocytes, the number of metaphase II oocytes, the percentage of CIVF or ICSI cycle and the number of embryos. The number of embryos transferred, clinical pregnancy rate, implantation rate, twin pregnancy rate, delivery rate, the number of embryos frozen, the distribution between day 2 and day 3 embryos frozen and the distribution between good quality day 2 and day 3 embryos frozen after fresh embryo transfer, mean number of thawed embryos, survival rate after thawing and mean number of embryos developed to blastocysts (days 5 and 6) were also comparable between the two groups (data not shown).
Table 2 summarizes the outcomes of embryo transfer. Morphology scores of blastocysts and mean number of blastocysts transferred were similar between the two groups. On the other hand, clinical pregnancy and implantation rates were significantly higher in the half opening group compared with the one eighth opening group ( $74 \%$ versus $43 \%, \mathrm{P}=0.0090 ; 52 \%$ versus $27 \%, \mathrm{P}=0.0117$ ). No differences in the twin pregnancy and miscarriage rates per clinical pregnancy were found between the two groups.

\section{Discussion}

Our results suggest that the size of zona pellucida thin- 
ning area or opening area can affect the outcome of cryopreserved embryo transfer and that higher pregnancy and implantation rates can be attained as the size of zona pellucida thinning area or opening area increases up to half.

Lyu et al. reported that when the slit size after assisted hatching for fresh human blastocysts was about one fifth of zona pellucida, significantly more of the larger inner cell mass became trapped by the zona pellucida opening during hatching than the smaller inner cell mass ${ }^{7)}$. They also reported that all the human blastocysts completely hatched after creating the slit with size beyond one third of zona pellucida. Therefore, it is suggested that quarter of zona pellucida thinning at the cleavage-stage or one eighth of zona pellucida opening at the blastocyst-stage of cryopreserved embryo transfer is inadequate for the completion of hatching process in some cases, however, it can be considerably improved by half of zona pellucida thinning or opening.

It is difficult to achieve half of zona pellucida thinning or opening with the use of an acid solution and the chemical needs to be washed out to prevent any damage to the embryo. The laser offers many advantages over traditional methods in a clinical situation where half of zona pellucida needs to be thinned or opened. However, this procedure may pose potential harm to embryos by heat shock. Hartshorn et al. demonstrated that mouse embryos at the eight-cell stage are able to respond to thermal shock by activating heat shock protein (hsp) production, as shown by the sharp increase in hsp70i transcription that follows embryo exposure to elevated temperature ${ }^{8}$ ). They also reported that in eight-cell mouse embryos, the procedure of all of zona pellucida opening with laser did

\section{References}

1: Andersen AN, Goossens V, Ferraretti AP, Bhattacharya S, Felberbaum R, de Mouzon J, and Nygren KG (2008): Assisted reproductive technology in Europe, 2004: results generated from European registers by ESHRE. Human Reproduction, 23:756 771 .

2: Mantoudis E, Podsiadly BT, Gorgy A, Venkat G, and Craft IL (2001): A comparison between quarter, partial and total laser assisted hatching in selected infertility patients. Hum Reproduction, 16:2182 2186 .

3: Kuwayama M, Vajta G, Ieda S, and Kato O (2005): Comparison of open and closed methods for vitrifi- not stimulate hsp70i expression in all blastomeres ${ }^{9)}$. In addition, our results for half of zona pellucida thinning or opening groups by laser demonstrated higher pregnancy and implantation rates without increasing the miscarriage rate compared with quarter thinning or opening groups. Moreover, Kanyo and Konc reported that there was no evidence of an increase in chromosomal aberrations or congenital malformations for 134 children born after laser assisted hatching 10). However, the fact that a pregnancy is established does not preclude that there are other underlying anomalies. Therefore, further long-term studies, such as looking at the birth defects and other anomalies that can occur later, are needed to confirm and assess the safety of half of zona pellucida thinning or opening by laser assisted hatching.

\section{Conclusions}

In cryopreserved cleaved embryo transfers, half of zona pellucida thinning at the cleavage-stage or half of zona pellucida opening at the blastocyst-stage by laser assisted hatching could significantly increase the rates of clinical pregnancy and implantation compared with quarter of zona pellucida thinning or one eighth opening. If the safety of laser assisted hatching is confirmed, half of zona pellucida thinning at the cleavage-stage or half of zona pellucida opening at the blastocyst-stage by laser assisted hatching should be performed routinely in cryopreserved cleaved embryo transfers. The laser seems to be beneficial, allowing for quick, easyto-perform in a clinical situation where half of zona pellucida needs to be thinned or opened in assisted reproductive technology.

cation of human embryos and the elimination of potential contamination. Reproductive BioMedicine Online, 11:608 614

4: Hiraoka K, Hiraoka K, Horiuchi T, Kusuda T, Okano S, Kinutani M, and Kinutani K (2009): Impact of the size of zona pellucida thinning area on vitrified-warmed cleavage-stage embryo transfers: a prospective, randomized study. Journal of Assisted Reproduction and Genetics, 26:515 521.

5: Gardner DK, and Schoolcraft WB: In-vitro culture of human blastocysts. In: (Jansen R, and Mortimer D, eds.) Towards reproductive certainty: fertility and genetics beyond 1999. 1999, Parthenon Press, 


\section{ORIGINAL ARTICLES}

London. pp $378 \sim 388$.

6: Hiraoka K, Fuchiwaki M, Hiraoka K, Horiuchi T, Murakami T, Kinutani M, and Kinutani K (2008): Effect of the size of zona pellucida opening by laser assisted hatching on clinical outcome of frozen cleaved embryos that were cultured to blastocyst after thawing in women with multiple implantation failures of embryo transfer: a retrospective study. Journal of Assisted Reproduction and Genetics, 25:129 135.

7: Lyu QF, Wu LQ, Li YP, Pan Q, Liu DE, Xia K, Liang DS, Cai F, Long ZG, Dai HP, and Xia JH (2005): An improved mechanical technique for assisted hatching. Human Reproduction, 20:1619
1623.

8: Hartshorn C, Anshelevich A, and Wangh LJ (2005): Rapid, single-tube method for quantitative preparation and analysis of RNA and DNA in samples as small as one cell. BMC Biotechnology, 13;5:2.

9: Hartshorn C, Anshelevich A, and Wangh LJ (2005): Laser zona drilling does not induce hsp70i transcription in blastomeres of eight-cell mouse embryos. Fertility and Sterility, 84:1547 1550.

10: Kanyo K, and Konc J (2003): A follow-up study of children born after diode laser assisted hatching. European journal of obstetrics, gynecology, and reproductive biology, 110:176 180. 